02,05

\title{
Размерные зависимости магнитных свойств сверхпроводящих наноструктур свинца в пористом стекле
}

\author{
( Н.Ю. Михайлин ${ }^{1}$, Д.В. Шамшур ${ }^{1}$, Р.В. Парфеньев ${ }^{1}$, В.И. Козуб ${ }^{1}$, \\ Ю.М. Гальперин ${ }^{1,2}$, Ю.А. Кумзеров ${ }^{1}$, А.В. Фокин ${ }^{1}$ \\ ${ }^{1}$ Физико-технический институт им. А.Ф. Иофрфе РАН, \\ Санкт-Петербург, Россия \\ ${ }^{2}$ Department of Physics, University of Oslo, \\ PO Box 1048 Blindern, 0316 Oslo, Norway \\ E-mail: mikhailin.nik@gmail.com
}

Представлены результаты изучения сверхпроводящих наноструктур, созданных путем заполнения из расплава под давлением матрицы пористого стекла свинцом (Pb-ПC). Изучены образцы с характерным диаметром пористой структуры $d \approx 7,3$ и $2 \mathrm{~nm}$. Обнаружено, что критическая температура сверхпроводящего перехода исследованных образцов близка к соответствующему значению для объемного свинца $T_{c} \approx 7.2 \mathrm{~K}$. В то же время, в нанокомпозитах обнаружено многократное увеличение критического магнитного поля вплоть до $H_{c}(T=0 \mathrm{~K})(\mathrm{Pb}-П \mathrm{C} 3 \mathrm{~nm}) \approx 165 \mathrm{kOe}$ по сравнению с $H_{c}(0)=803$ Ое для массивного $\mathrm{Pb}$. Низкотемпературные магнитополевые зависимости магнитного момента $M(H)$ характеризуются наличием квазипериодических скачков потока, исчезающих по мере уменьшения диаметра свинцовых наноструктур. Рассматривается качественная модель наблюдаемых эффектов.

DOI: 10.21883/FTT.2018.06.45976.11M

\section{Введение}

Исследование сверхпроводящих (СП) наноструктур представляет большой интерес, так как в таких структурах физические свойства, в том числе параметры СП-состояния, меняются в зависимости от размера и связности СП-структуры. СП-состояние в образцах, характерные размеры которых сравнимы с длиной когерентности $\xi$ и глубиной проникновения магнитного поля $\lambda$, может существенно отличаться от СП-состояния объемных материалов.

В настоящей работе представлены результаты изучения СП-наноструктур, созданных путем заполнения из расплава под давлением матрицы - пористого стекла (ПС) - СП-металлом (свинцом). Достоинством этого способа наноструктурирования по сравнению с другими (напыление сверхтонких пленок, создание структур методом нанолитографии, использование в качестве матрицы искусственного опала и др.) состоит в том, что таким образом можно создавать трехмерные массивы наночастиц с различными характерными диаметрами вплоть до $d \sim 2 \mathrm{~nm}$. В качестве матрицы использовалось натрий-боросиликатное стекло ДВ1, в котором при химической обработке и отжиге образовывалась система хаотично пересекающихся пор с малым разбросом $(\sim 5 \%)$ по диаметру $d$ (рис. $1, a, b)$, объем системы пор составлял $\sim 25 \%$ общего объема стекла [1]. Для исследования были отобраны образцы с $d \approx 7,3$ и $2 \mathrm{~nm}$. Отметим, что механическая прочность нанокомпозитов заметно уменьшалась по мере уменьшения характерного диаметра токопроводящей сети. Заполнение пор свинцом составляло $\sim 95 \%$

\section{Экспериментальные результаты и их обсуждение}

Исследования структур подобного типа, созданных при введении индия в поры опала с минимальными размерами $d \geq 10 \mathrm{~nm}$, показали, что сетка СП-частиц обладает свойствами, характерными для СП-материала 2 рода [2]. Также в них наблюдается рост значений критического магнитного поля СП-перехода $\left(H_{c} \leq 2 \mathrm{~T}\right.$ при $T=0 \mathrm{~K})$ и критической температуры $\left(T_{c} \leq 4.5 \mathrm{~K}\right)$ по сравнению со значениями для массивного индия $\left(T_{c}=3.4 \mathrm{~K}, H_{c}(0)=280 \mathrm{Oe}\right)$. Аналогичные эффекты изучались также в различных структурах на основе In $[2,3]$ и $\mathrm{Pb}[4,5]$. Отметим, что для массивного свинца $T_{c}=7.2 \mathrm{~K}$, и заметного роста $T_{c}$ в структурах на основе свинца, как правило, не наблюдалось. Ширина СП-перехода в наноструктурах, определенная из зависимостей сопротивления $R$ от температуры $R(T)$ и от магнитного поля $R(H)$, увеличивалась по сравнению с объемным металлом.

Магнитные свойства пористых матриц, заполненных СП I рода, существенно отличаются от магнитных свойств массивного материала. Зависимость магнитного момента от магнитного поля $M(H)$ характеризуется большим гистерезисом и наличием скачков $M$ в зависимости от внешнего магнитного поля при $T<T_{c}[5,6]$.

Магнитополевые и температурные зависимости магнитного момента нанокомпозитов $\mathrm{Pb}-П \mathrm{C}$ с характерным диаметром пор $7 \mathrm{~nm}$ (изучался в виде порошка и объемного материала), $3 \mathrm{~nm}$ (объем) и $2 \mathrm{~nm}$ (объем) были измерены в диапазоне температур $T=2 \mathrm{~K}-7 \mathrm{~K}$ в магнитном поле до $140 \mathrm{kOe}$ с помощью вибрационного магнитометра, в том числе на установке PPMS-14. На 

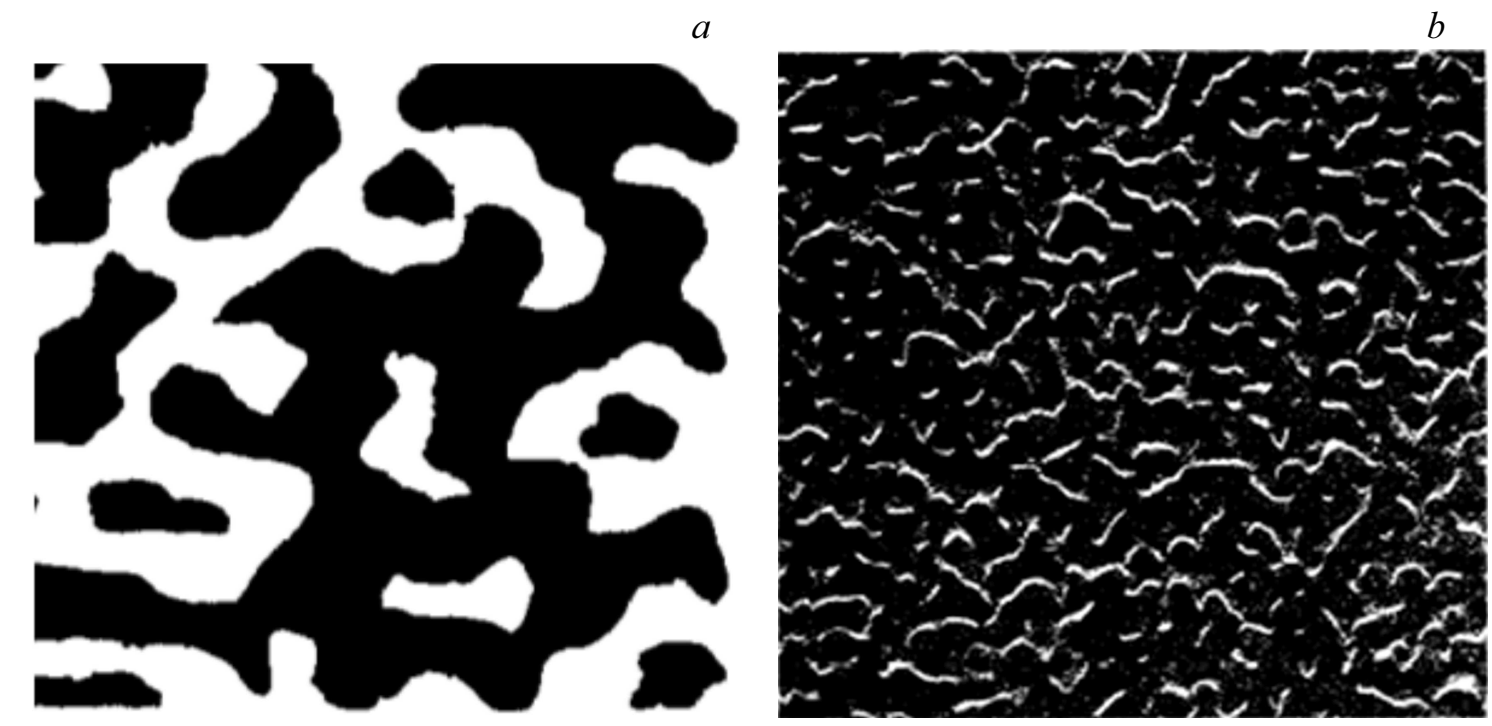

$1 \mu \mathrm{m}$

Рис. 1. Слева: схематическое изображение структуры пористого стекла (светлый контраст - поры, в дальнейшем заполняемые СП-металлом; справа: фотография пористого стекла [1]).

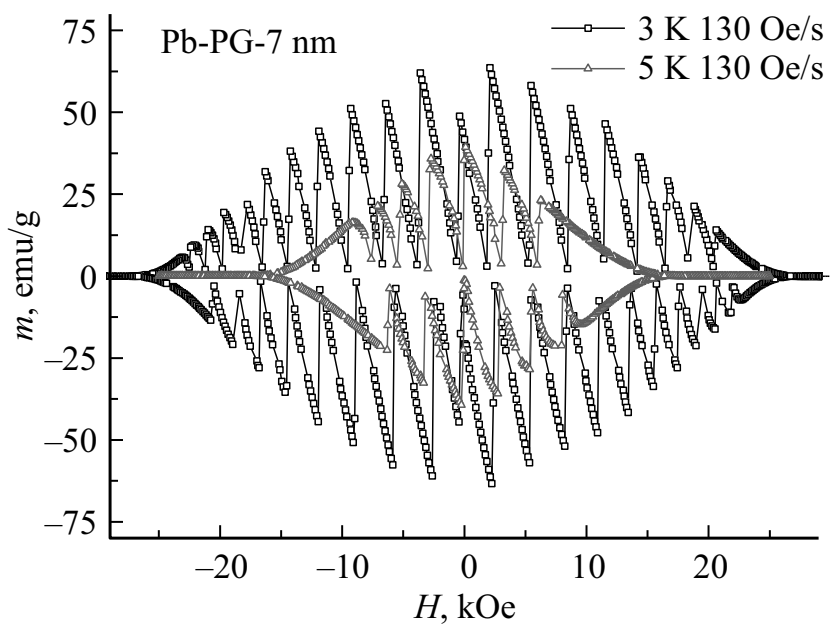

Рис. 2. Зависимость намагниченности $m$ нанокомпозита $\mathrm{Pb}-\Pi \mathrm{C}(7 \mathrm{~nm}$, объем $)$ от магнитного поля $H$, скорость развертки магнитного поля $d H / d t=130 \mathrm{Oe} / \mathrm{sec}, T=3 \mathrm{~K}, 5 \mathrm{~K}$.

рис. 2 показана зависимость намагниченности $m$ (магнитный момент, отнесенный к единице массы) нанокомпозита $\mathrm{Pb}-\Pi \mathrm{C}$ ( $7 \mathrm{~nm}$, объем) $m(H)$ в магнитном поле $H$ при различных температурах $T=3$ и $5 \mathrm{~K}$. Зависимость имеет сложный вид с гистерезисом и наличием скачков $m(H)$, число и положение которых зависит от температуры. Как видно из зависимости на рис. 2 , в $\mathrm{Pb}-$ ПС $(d \approx 7 \mathrm{~nm})$ уже при $T=3 \mathrm{~K}$ достигается $H_{c} \leq 27 \mathrm{kOe}$, что многократно превышает значения для массивного свинца $H_{c}(T=0 \mathrm{~K})=803 \mathrm{Oe}$.

Измерения намагниченности нанокомпозита $\mathrm{Pb}-П \mathrm{C}$ $7 \mathrm{~nm}$ в виде массивной частицы и порошка проводились с целью изучения влияния макроскопической структуры образца на лавинообразные процессы, происходящие во время скачка намагниченности. В массивном образце лавинообразные процессы развиваются по всему объему, и, как следствие, в нем наблюдаются ярко выраженные скачки намагниченности. В порошке границы и пустоты между отдельными частицами могли бы препятствовать развитию скачка, в результате чего на зависимости наблюдалось бы большое количество скачков с малой амплитудой, соответствующих отдельным частицам в порошке. Оказалось, однако, что структура скачков в случае объемного нанокомпозита $\mathrm{Pb}-\Pi \mathrm{C}$ и порошка с диаметром частиц $D \sim 100 \mu \mathrm{m}$ близка, как следует из экспе-

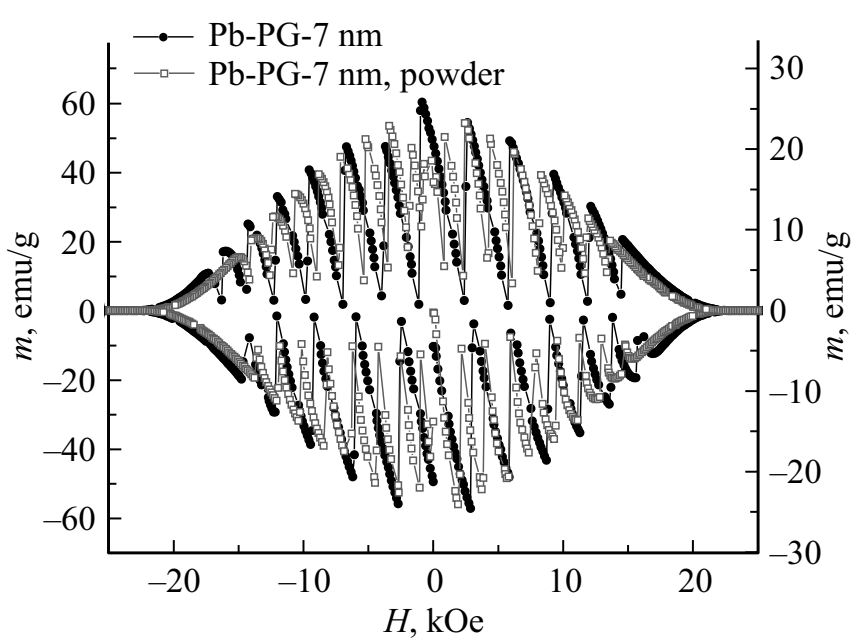

Рис. 3. Сравнение магнитополевой зависимости намагниченности $m(H)$ образцов нанокомпозита $\mathrm{Pb}-\Pi \mathrm{C}(7 \mathrm{~nm}$, объем) и $\mathrm{Pb}-\Pi \mathrm{C}(7 \mathrm{~nm}$, порошок), скорость развертки магнитного поля $d H / d t=130 \mathrm{Oe} / \mathrm{sec}, T=4 \mathrm{~K}$. 


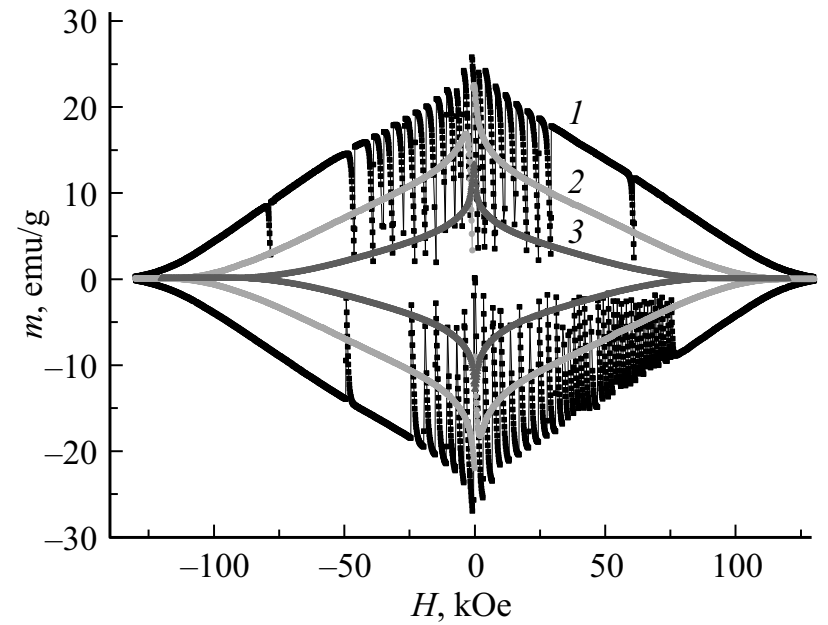

Pис. 4. Зависимость магнитного момента $M$ нанокомпозита $\mathrm{Pb}-\Pi \mathrm{C}(3 \mathrm{~nm})$ от магнитного поля, скорость развертки магнитного поля $d H / d t=130 \mathrm{Oe} / \mathrm{sec}, T=2 \mathrm{~K}(1), 3 \mathrm{~K}(2), 4 \mathrm{~K}$ (3).

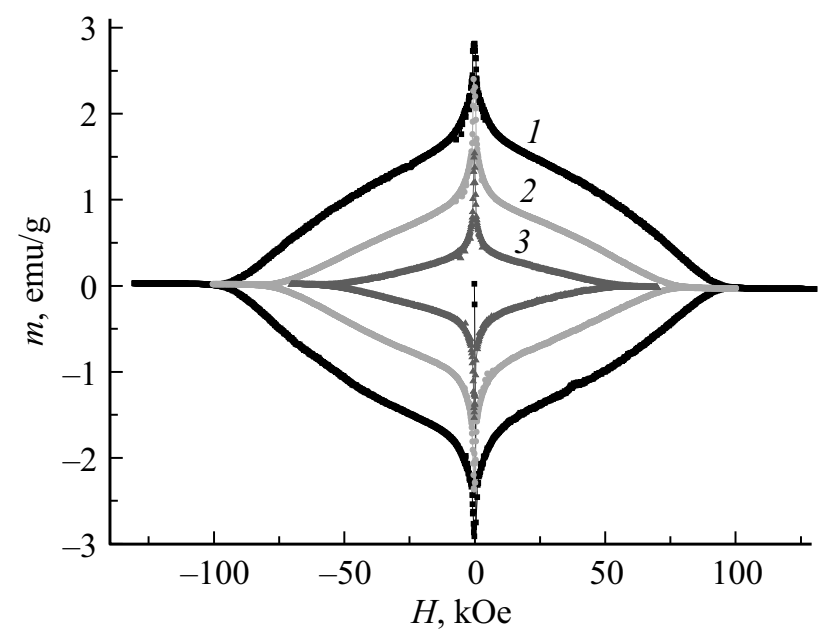

Рис. 5. Зависимость намагниченности $m$ нанокомпозита $\mathrm{Pb}-\Pi \mathrm{C}(2 \mathrm{~nm})$ от магнитного поля при различных температурах $T=2 K(1), 3 \mathrm{~K}(2)$ и $4 \mathrm{~K}$ (3). Скорость развертки магнитного поля $d H / d t=130 \mathrm{Oe} / \mathrm{sec}$.

риментальных зависимостей на рис. 3, где показано сравнение магнитополевых зависимостей намагниченности $\mathrm{m}(\mathrm{H})$ образцов нанокомпозита $\mathrm{Pb}-\Pi \mathrm{C}(7 \mathrm{~nm}$, объем) и $\mathrm{Pb}-\Pi \mathrm{C}(7 \mathrm{~nm}$, порошок) при одинаковых условиях (скорость развертки магнитного поля $130 \mathrm{Oe} / \mathrm{sec}, T=4 \mathrm{~K}$ ).

На рис. 4 показано, как уменьшение диаметра токопроводящей сетки в $\mathrm{Pb}-\Pi \mathrm{C}(d \approx 3 \mathrm{~nm})$ отражается на изменении характера скачков магнитного момента $M(H)$ и температуре их появления $(T \leq 3 \mathrm{~K})$. Качественно зависимости $M(H)$ подобны, различия наблюдаются в величине $H_{c}$, температуре появления скачков потока и их количестве. При сравнимой массе образцов $\mathrm{Pb}-$ ПС $7 \mathrm{~nm}$ и $\mathrm{Pb}-$ ПС $3 \mathrm{~nm}$ наблюдается кратное уменьшение величины магнитного момента в нанокомпозите $\mathrm{Pb}-$ ПС $(d \approx 3 \mathrm{~nm})$; одновременно критическое магнитное поле образца $H_{c}$ при $T=3 \mathrm{~K}$ достигает величины $128 \mathrm{kOe}$ по сравнению с $H_{c}=27 \mathrm{kOе}$ в образце с $d \approx 7 \mathrm{~nm}$.

На рис. 5 показаны зависимости магнитного момента $M$ нанокомпозита $\mathrm{Pb}-\Pi \mathrm{C}(2 \mathrm{~nm})$ от магнитного поля при различных температурах $T=2 \mathrm{~K}, 3 \mathrm{~K}$ и $4 \mathrm{~K} . H_{c}$ нанокомпозита, как и в предыдущих случаях, на порядки превышает $H_{c}$ массивного свинца и достигает при $T=3 \mathrm{~K}$ величины $H_{c}=110 \mathrm{kOe}$. Эта величина близка к соответствующей в образце $\mathrm{Pb}-\Pi \mathrm{C}(3 \mathrm{~nm})$ и также значительно больше $H_{c}$ нанокомпозита $\mathrm{Pb}-\Pi \mathrm{C}(7 \mathrm{~nm})$. В то же время обращает на себя внимание отсутствие скачков магнитного потока на зависимостях $M(T)$ в $\mathrm{Pb}-\Pi \mathrm{C}(2 \mathrm{~nm})$ при всех температурах вплоть до $T=2 \mathrm{~K}$ и при той же скорости развертки магнитного поля $d H / d t=130 \mathrm{Oe} / \mathrm{sec}$, что и в представленных ранее на pис. 2-4 зависимостях. Отметим также, что в $\mathrm{Pb}-$ ПС $(2 \mathrm{~nm})$ пиковое значение $m$ на порядок меньше, чем в $\mathrm{Pb}-\Pi \mathrm{C}(3 \mathrm{~nm})$.

Ранее скачки магнитного потока наблюдались в индиевых наноструктурах на основе искусственного опала [6], свинцовых наноструктурах в пористом стекле $(d \approx 7 \mathrm{~nm})$ [7], а также в объемных СП 2-го рода [8]. В последнем случае их появление связывают с термомагнитной неустойчивостью системы вихрей, когда срыв нескольких вихрей с центров пиннинга вызывает локальное выделение тепла, которое приводит к локальному повышению температуры и в свою очередь ослабляет пиннинг вихрей. В результате создается положительная обратная связь, и происходит лавинообразное проникновение магнитного поля в материал.

В наноструктурах также реализуется механизм, связанный с локальным нагревом, который посредством положительной обратной связи вызывает рост температуры всего образца, однако его природа, по-видимому, другая. Характерный диаметр свинца в изученных наноструктурах много меньше глубины проникновения магнитного поля $\lambda$ в объемном свинце $(\lambda(0 K)=39 \mathrm{~nm})$, и в нитях свинца вихри образоваться не могут. В то же время в нанокомпозитах имеется многосвязная система СП-контуров, и при увеличении $H$, когда достигается критический ток для одного или нескольких контуров, происходит скачкообразное проникновение магнитного потока в них, сопровождающееся выделением тепла. Можно предположить, что „спусковым механизмом“ при возникновении скачков магнитного потока в нанокомпозитах являются случайные слабые связи в СП-контурах.

Как видно из рис. 3-5, величина максимума магнитного момента $H$ на зависимости $M(H)$ падает с уменьшением характерного размера элемента структуры.Как уже отмечалось, с уменьшением характерного диаметра $d$ СП-нанокомпозита его механическая прочность также уменьшается. Можно предположить, что при использовании матрицы с меньшим размером пор ухудшается однородность внутренней структуры нанокомпозита, т.е. увеличивается количество слабых связей и уменьшается их „однородность“. Это может служить причиной того, что скачки магнитного потока 


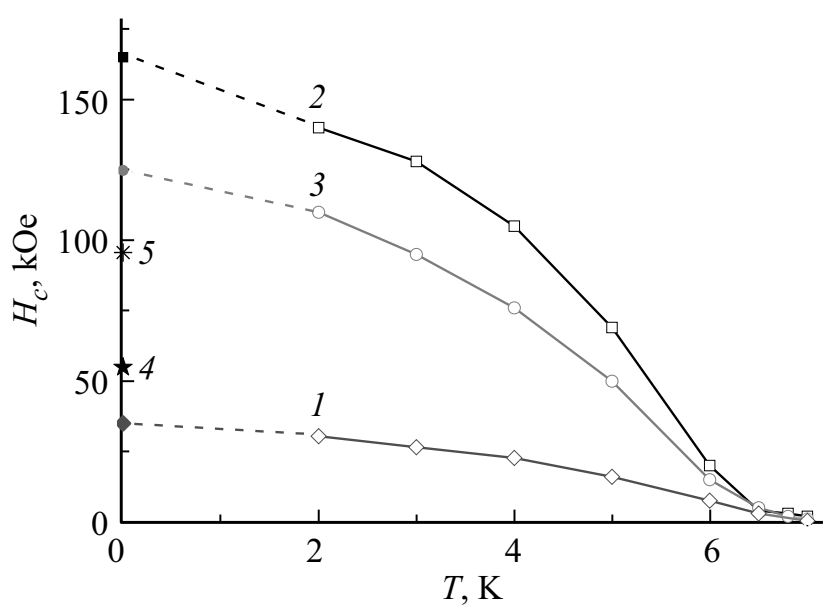

Рис. 6. Зависимость критического магнитного поля в нанокомпозитах $\mathrm{Pb}-\Pi \mathrm{C}, d \approx 7 \mathrm{~nm}$ (1, порошок и объем), $3 \mathrm{~nm}(2)$ и $2 \mathrm{~nm}(3)$, от температуры. Также приведены данные [8] для $\mathrm{Pb}-$ ПС $3 \mathrm{~nm}(4)$ и $6 \mathrm{~nm}(5)$.

в $\mathrm{Pb}-$ ПС по мере уменьшения $d$ проявляются при все более низких температурах, и в нанокомпозите с $d \approx 2 \mathrm{~nm}$ не видны вплоть до $T=2 \mathrm{~K}$, как следует из данных рис. 2, 4, 5. Учитывая, что намеренное нарушение структуры образца $\mathrm{Pb}-\Pi \mathrm{C} d \approx 7 \mathrm{~nm}$ вплоть до порошка с диаметром частиц $D \sim 100 \mu \mathrm{m}$ не привело к изменению характера скачков $m(H)$ (см. рис. 3 ), следует заключить, что в нанокомпозитах $\mathrm{Pb}-\Pi \mathrm{C} d \approx 3 \mathrm{~nm}$, $2 \mathrm{~nm}$ неоднородности СП-сети проявляются на заметно меньших масштабах.

Из зависимостей $m(H, T)$ и $M(H, T)$ были определены величины критических магнитных полей $H_{c}(T)$ в нанокомпозитах $\mathrm{Pb}-\Pi \mathrm{C}, 2,3$ и $7 \mathrm{~nm}$ (рис. 6) (отметим, что значения для массивного образца $\mathrm{Pb}-\Pi \mathrm{C}, d \approx 7 \mathrm{~nm}$, и порошка из того же материала практически совпадают, как показано на рис. 4). Критическое поле в образце $\mathrm{Pb}-П \mathrm{C}(3 \mathrm{~nm})$ достигает $H_{c}>140 \mathrm{kOе}$ при $T=2 \mathrm{~K}$, что более чем на 2 порядка превышает критическое поле массивного свинца, и в $\sim 5$ раз значения, полученные в $\mathrm{Pb}-\Pi \mathrm{C}, 7 \mathrm{~nm}\left(H_{c} \approx 30 \mathrm{kOe}\right.$ при $\left.T=2 \mathrm{~K}\right)$. Дальнейшее уменьшение характерного диаметра свинцового нанокомпозита до $d \approx 2 \mathrm{~nm}$ не оказывает существенного влияния на $H_{c} \approx 110 \mathrm{kOe}$ при $T=2 \mathrm{~K}$. Заметим, что полученные нами результаты для $\mathrm{Pb}-\Pi \mathrm{C}(3 \mathrm{~nm})$ в $\sim 1.5$ раза превышают данные [8]. Отметим также, что в экспериментальных данных, приведенных на рис. 6, обращает на себя внимание изменение вида зависимости $H_{c}(T)$ при $T$ близких к $T_{c}$.

Таким образом, исследованные образцы демонстрируют необычайно высокие значения критического магнитного поля. $H_{c}(0)$, определенное экстраполяцией по формуле $H_{c}(T)=H_{c}(0)\left(1-\left(T / T_{c}\right)^{2}\right)$, увеличивается от $H_{c}(0)(\mathrm{Pb}-\Pi \mathrm{C}(7 \mathrm{~nm}$, порошок и объем $)) \approx 35 \mathrm{kOe}$ до $H_{c}(0)(\mathrm{Pb}-\Pi \mathrm{C}(3 \mathrm{~nm})) \approx 165 \mathrm{kOе}$. Отметим, что в рассматриваемых нанокомпозитах $\mathrm{Pb}-$ ПС длина свободного пробега ограничена поперечным размером нано- структур свинца $d \geq 2 \mathrm{~nm}$; глубина проникновения магнитного поля $\lambda \gg d$ (для массивного свинца $\lambda \approx 39 \mathrm{~nm}$ при $T=0 \mathrm{~K}$ ). В соответствии с этим в изученных образцах $\mathrm{Pb}-П \mathrm{C}$ должен проявляться размерный эффект увеличения критического магнитного поля $H_{c}$, что и наблюдается в эксперименте (рис. 6). Оценка верхнего критического магнитного поля была выполнена в соответствии с формулой [9] для СП-цилиндра в магнитном поле $H_{c}^{d}(T)=\kappa \cdot H_{c}^{\text {bulk }}(T) \cdot \lambda(T) / d$. Выбрав коэффициент $\kappa=6$ (промежуточное значение между $\kappa=4$ и $\kappa=8$ в случае перпендикулярного и параллельного направления $H$ относительно оси цилиндра) и используя $\lambda \approx 39 \mathrm{~nm}$ (для объемного свинца), получим $H_{c}^{d}(\mathrm{~Pb}-$ ПС $7 \mathrm{~nm}) \approx 27 \mathrm{kOe}$ и $H_{c}^{d}(\mathrm{~Pb}-$ ПС $2 \mathrm{~nm}) \approx 94 \mathrm{kOe}$, что удовлетворительно согласуется с полученными экспериментально значениями.

\section{Заключение}

В работе представлены результаты изучения низкотемпературных магнитных свойств СП-наноструктур, созданных путем заполнения пористого стекла (диаметр пор $d \approx 7,3$ и $2 \mathrm{~nm}$ ) свинцом. Установлено, что критическая температура СП-перехода нанокомпозитов $T_{c} \approx 7.2 \mathrm{~K}$, что хорошо совпадает с $T_{c}$ массивного свинца. В то же время, при уменьшении характерного диаметра проводящей сети $d$ в изученных образцах наблюдается многократное возрастание критического магнитного поля вплоть до $H_{c}(0)(\mathrm{Pb}-\Pi \mathrm{C}(3 \mathrm{~nm})) \approx 165 \mathrm{kOe}$, что удовлетворительно согласуется с теоретическими оценками для верхнего критического поля с учетом размерного эффекта. Низкотемпературные магнитополевые зависимости магнитного момента $M(H)$ характеризуются наличием квазипериодических скачков потока, исчезающих по мере уменьшения диаметра свинцовых наноструктур.

\section{Список литературы}

[1] H. Thomas. Elmer Corning, Incorporated, Reprinted with permission from „Porous and Reconstructed Glasses“. Engineered Materials Handbook. V. 4, Ceramic and Glasses. P427-32. Copyright 1932.

[2] Д.В. Шамшур, А.В. Черняев, А.В. Фокин, С.Г. Романов. ФТТ 47, 11, 1927 (2005).

[3] M.J. Graf, T.E. Huber, C.A. Huber. Phys. Rev. B 45, 6 (1992).

[4] A.E. Aliev, S.B. Lee, A.A. Zakhidov, R.H. Baughman. Physica C 453, 15 (2007).

[5] C. Tien, E.V. Charnaya, D.Y. Xing, A.L. Pirozerskii, Yu.A. Kumzerov, Y.S. Ciou, M.K. Lee. Phys. Rev. B 83, 014502 (2011).

[6] R.V. Parfeniev, D.V. Shamshur, M.S. Kononchuk, A.V. Chernyaev, S.G. Romanov, A.V. Fokin. Proc. 24 Int. Conf. on Low Temp. Physics. Orlando, Florida, USA. August 10-17, 235 (2005).

[7] K.-H. Miiller, C. Andrikidis. Phys. Rev. B 49, 2, 1294 (1994-II).

[8] N.K. Hindlev, J.H.P. Watson. Phys. Rev. 183, 2, 525 (1969).

[9] В.Л. Гинзбург. ЖЭТФ 34, 113 (1958). 